\title{
Inhibitory Effect of Nicotine on Apoptosis Induced by Endoplasmic Reticulum Stress
}

\author{
Dong-Hee LEE \\ Department of Life Sciences, University of Seoul, 90 Jeonnong-Dong, Seoul, Korea 130-743
}

(Received August 10, 2007; Accepted December 14, 2007)

\begin{abstract}
Cigarette smoking causes serious health problems in humans, especially if smoking habits are established during their adolescence. Nicotine is known to mutate DNA and interfere with apoptosis. Apoptosis is considered as a potent defense mechanism against cellular damaging agents. This study aims to investigate the effect of nicotine on the progression of apoptosis induced under ER stress conditions using four different established cell lines: HEK293, 3T3-L1, C2C12, and HepG2. When treated with nicotine, the progression of apoptosis was notably inhibited in the four cell lines according to the assays of caspase-3 activation and DNA fragmentation. In ERstressed cells, nicotine appears to inhibit the progression of apoptosis in a concentration-dependent manner. When cells were treated with nicotine prior to ER stress, GRP94 level significantly increased compared to other ER stress markers of PDI and GRP78. This observation suggests that the inhibitory effect of nicotine may results from up-regulation of GRP94, an anti-apoptotic chaperone, under nicotine treatment. Taken together, the present study strongly implies that nicotine may inhibit apoptosis, caused by prolonged ER stress, based on promotion of GRP94 expression.
\end{abstract}

Keywords $\square$ apoptosis, endoplasmic reticulum stress, nicotine, caspase-3, TUNEL

\section{INTRODUCTION}

Cigarette smoke contains numerous hazardous components, among which nicotine accounts for many types of circulatory and gastrointestinal disorders and, especially, for lung cancer (Blackburn et al., 1997; Chung et al., 1999). In wake of health warnings against smoking, the number of smokers gradually decreases on the global basis; however, that of underage or woman smokers have sharply increased over last decade. According to recent reports, at least one quarter of US teenagers are considered as habitual smokers.

Underage smokers suffer long lasting DNA damage in comparison to those who start smoking after adolescence. In addition, underage smoking is very serious since juvenile smokers easily develop severe addiction symptoms to nicotine, very different from post-adolescent beginners (Fujiki et al., 1998; Gupta et al., 1996; Schuller et al., 1995). Indeed, the underage smokers frequently suffer various cardiovascular and pulmonary disorders and even cancers in their later stages of life.

*Corresponding author

Tel: 02-2210-2170,

Fax: $02-2210-2888$
According to the Journal of American Cancer Society, smokers below 18 years old most likely encounter with permanent mutations in their genome and they are at greater risk for cancer even though they quit smoking in a few years (Fassina et al., 2004; Prokopczyk et al., 1997). Nicotine is considered not only as a xenotoxic agent to damage the integrity of DNA but as interfering with the cellular apoptotic pathway. Apoptosis serves as a voluntary defense mechanism against cellular damaging agents (Chin et al., 2007). Under circumstances, cells harboring damaged DNAs may escape from the apoptotic pathway, survive and further proliferate in the presence of cellular nicotine (Mussman et al., 2007; Karla et al., 2000). When apoptosis is inhibited for underage smokers due to nicotine's interference with apoptosis, the likelihood of suffering cancers is relatively high throughout their life period.

In the present study, the effect of nicotine on the progression of apoptosis was investigated under endoplasmic reticulum stress (ERS) conditions. Apoptosis is usually evident under prolonged ERS. In four different established cell lines (HEK293, 3T3-L1, C2C12, and HepG2), ERS was induced by tunicamycin, which is known a powerful ERS inducer according to its blockage of post-translational modification (Karaskov et al., 2007; Yung et al., 2007). The progression of and ERS 
and ensuing apoptosis was significantly inhibited in the four cell lines. Based on the assays of caspase-3 activation and DNA fragmentation, nicotine evidently exerts an inhibitory effect on the progression of apoptosis.

Treatment with nicotine concurred with up-regulation of GRP94, an anti-apoptotic molecular chaperone, in this study. The increment of GRP94 may be due to nicotine treatment. When the cells were treated with nicotine prior to ER stress, the level of GRP94 was significantly increased compared to other ER stress markers of PDI and GRP78. This significant up-regulation of GRP94 suggests that nicotine may retard apoptosis induced under prolonged ER stress by enhancing GRP94 expression.

\section{MATERIALS AND METHODS}

\section{Cell culture}

Four different cell lines were used in this study: HEK293, HepG2, 3T3-L1 (murine preadipocyte), and C2C12 (murine premyocyte). Cells were cultured for a week with DMEM containing 5\% FBS. Once the cells reached full confluency, they were divided into aliquots and further cultured in media containing 0-5 mM tunicamycin for 2 hours. The tunicamycintreated cells were later cultured with nicotine during various periods (0-120 minutes). Following treatment with nicotine during $0,10,20,30,60$, or 120 minutes, the cells were washed three times with PBS. Nicotine was purchased from Sigma Chemical Company (St. Louis, MO, USA) and delivered into media as dissolved in $95 \%$ ethanol. To study whether nicotine affects the expression of molecular chaperones, cells were pretreated with nicotine before they were later treated with 2.5 $\mathrm{mM}$ tunicamycin for one hour.

\section{TUNEL assay}

DNA fragmentation assay was performed by terminal deoxyribonucleotide transferase (TdT)-mediated dUTP nick-end labeling (TUNEL) using the In Situ Cell Death Detection Kit (Boehringer-Mannheim) according to the manufacturer's protocol. For TUNEL assay, cells were treated with nicotine at 37 ${ }^{\circ} \mathrm{C}$ for 2 hours. After treatments, the cells were treated with tunicamycin at various concentrations and fixed in $4 \%$ paraformaldehyde, and blocked with hydrogen peroxide in methanol distilled deionized water. TUNEL-positive cells were detected on the basis of brown nuclear stain and altered nuclear morphology. The extent of DNA strand breakage was measured using DNase-treated samples as positive controls. The ratio of TUNEL positive cells was calculated by the fraction of TUNEL-positive cells among 500 cells counted. ER stressinduced apoptosis was investigated when cells were treated with $1 \mathrm{mM}$ tunicamycin for 0-24 hours.

\section{Western Blotting and Caspase-3 quantitation}

Molecular chaperone expression was detected using rabbit antisera against GRP78, GRP94, and PDI (Santa Cruz Biotechnology, USA). The extent of apoptosis was assayed via the activation of endogenous caspase- 3 using antisera against the activated form. This assay measures the amount of activated caspase- 3 in response to the addition of tunicamycin. Cell lysates were prepared as $2 \times 10^{6}$ cells per $\mathrm{ml}$ at each time point after treatment. Fluorescence intensities of labelled antibodies are measured using a Gemini XPS Microplate Reader (Molecular Devices, Sunnyvale, USA).

\section{RESULTS}

Treatment of HEK293 with tunicamycin caused a concentration-dependent expression of the ER stress markers; however, no significant protein expression of the markers was observed in the absence of ER stress. Incubation of HEK293 with tunicamycin induced GRP78 expression. In response to tunicamycin $(0.5 \mathrm{mM})$, the expression of the molecular chaperones was notably greater than the response obtained in the absence of tunicamycin (Fig. 1). Up to $5 \mathrm{mM}$ of tunicamycin, the expression of GRP78 and GRP94 gradually increases in a concentration-dependent fashion. ERS-induced apoptosis, by tunicamycin, was supported by caspase-3 quantitation and TUNEL assays. Fig. 2 shows that the ratio of TUNEL-positive cells steadily increased up to $24 \mathrm{hrs}$ following treatment with tunicamycin at $2.5 \mathrm{mM}$. After treatment with tunicamycin, the TUNEL-positive ratio increased 7 times over the tunicamycin treatment for 24 hrs. No significant TUNEL staining, however, was shown to increase for the PBS-treated counterpart.

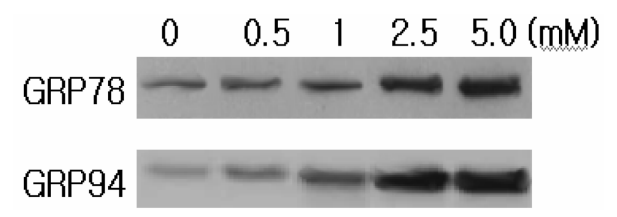

Fig. 1. Molecular chaperon expression under tunicamycin treatment. HEK293 cells were treated with tunicamycin depending on concentration (0-5 mM). The expression of GRP78 and GRP94 steadily increased in a concentration-dependent manner. 


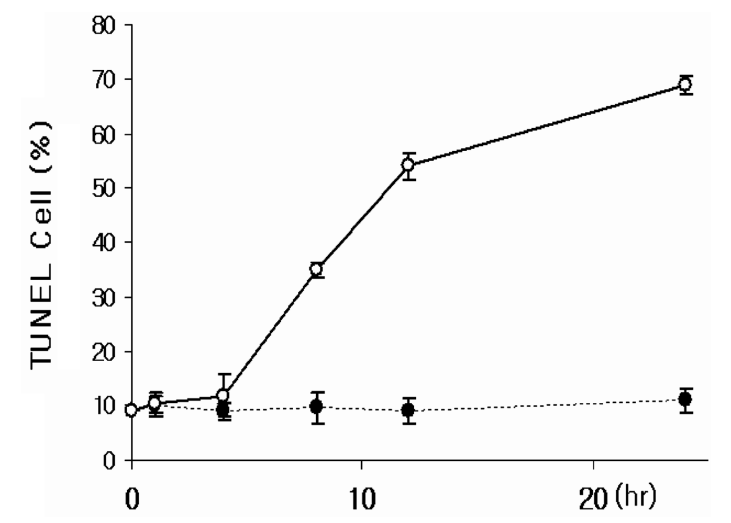

Fig. 2. TUNEL assay on ER stress-induced apoptosis. HEK293 cells were treated for various time periods with $2.5 \mathrm{mM}$ tunicamycin (open circle) or PBS (closed circle). TUNEL Cell (\%) refers to the percentage of TUNEL-positive cells representing apoptotic cells per 500 cell counts.

The effect of ERS on the cell lines was measured by caspase3 activation assay. We measured the degree of caspase-3 activation after addition of tunicamycin and incubation for 6 hrs. A time-dependent processing of caspase-3 was evident in response to tunicamycin treatment (Fig. 3). When HEK293 cells were exposed to $2.5 \mathrm{mM}$ tunicamycin, the presence of active form of caspase-3 was significantly higher compared to the control (PBS-treated counterpart). The data from this experiment indicate that the DNA fragmentation was significantly inhibited in the presence of $2 \mathrm{mM}$ nicotine. This result implies that the nicotine may play an important role in inhibiting the apoptotic progression which is triggered by tunicamycin.

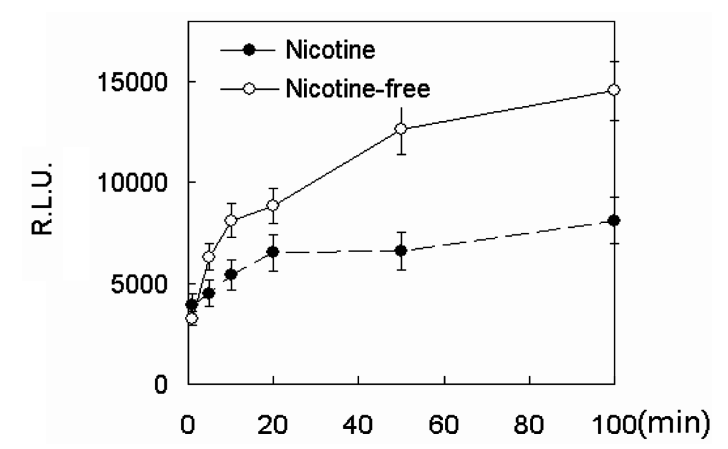

Fig. 3. Time course of caspase- 3 activation under nicotine treatment. Time-dependent processing of caspase-3 in ER stressed cells under nicotine treatment. HEK293 cells were exposed to tunicamycin $(2.5 \mathrm{mM})$ and further treated with nicotine for various periods and the presence of active form of caspase-3 was measured as described in Materials and Methods. RLU refers to relative luminescence unit.

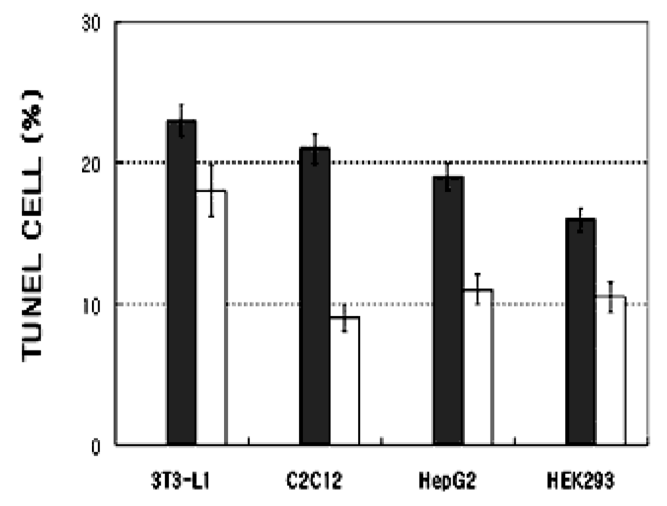

Fig. 4. Inhibition of Apoptosis induced by ER stress among cell lines. Count of TUNEL positive cell was made on the four lines of cell. Cells were treated only with $2.5 \mathrm{mM}$ tunicamycin (dark bars) and further incubated in the presence of $2 \mathrm{mM}$ nicotine (light bars). Error bars represent standard errors $(n=3)$. As in Fig. 2, TUNEL Cell (\%) refers to the portion of TUNELpositive cells per 500 cell counts.

Significant level of apoptosis was caused by tunicamycin (5 $\mathrm{mM}$ ) treatment in the four cell lines tested in this study. Irrespective of cell origin, the cell lines show inhibition of apoptosis in the presence of nicotine. Fig. 4 shows the typical outcomes of apoptosis and its inhibition according to the percentage of TUNEL-positive cells. Although a direct comparison may not be feasible between the cell lines, a consistent inhibitory pattern was obvious despite the different inhibitory effects of nicotine among the cell lines (Fig. 5). In working against apoptosis, the potential intracellular effectors might

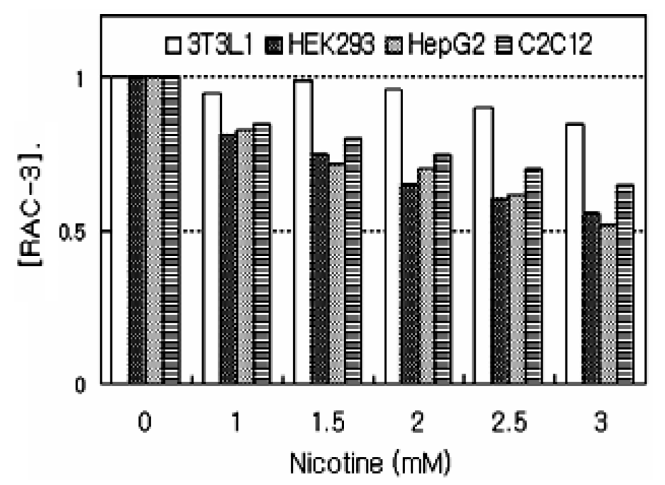

Fig. 5. Inhibition of apoptosis by nicotine concentration. Nicotine's inhibitory effect on apoptosis was quantitated via the presence of relative active form of capase-3. The extent of active caspase- 3 appears to decrease in response to the increment of nicotine concentration. In this experiment, [RAC-3] (relative active caspase-3) was calculated assuming the RLU of nicotine-free treatment is equal to 1.0 for each cell line. 


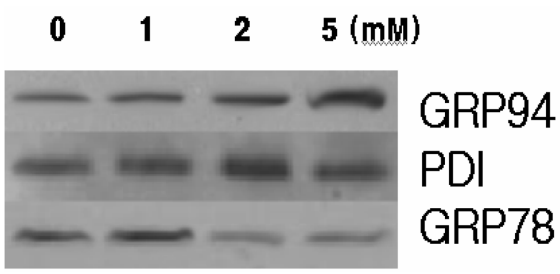

Fig. 6. Effect of nicotine on molecular chaperone expression. 3T3L1 cells were pre-treated with nicotine at $0-5 \mathrm{mM}$ and, later, subjected to ER stress conditions for $1 \mathrm{hr}$ by adding tunicamycin at $2.5 \mathrm{mM}$. Western blotting was performed using three respective antibodies. The nicotine pretreatment affects expressions of PDI and GRP78 minimally or negatively along the increment of nicotine concentration; however, GRP94 expression was consistently increased up to the highest nicotine concentration used in this experiment $(5 \mathrm{mM})$.

respond differently to the nicotine depending on the cellular background specific to each cell type. The relative active caspase- 3 measurement decreased as the amount of nicotine was reinforced up to $3 \mathrm{mM}$ in the media.

The inhibitory effect of nicotine may be due to up-regulation of GRP94, an anti-apoptotic chaperone, under nicotine treatment (Fig. 6). When the cells were treated with nicotine prior to ER stress, GRP94 level significantly increased compared to other ER stress markers of PDI and GRP78, whose presence remained steady or decreased against increased nicotine concentrations. Taken together, the present study strongly suggests that nicotine inhibits apoptosis which is induced under prolonged ER stress due to a potential up-regulation of GRP94.

\section{DISCUSSION}

Smoking has been implicated in a wide range of human diseases including heart malfunction, emphysema, and cancer. Nicotine is considered as the prime pathological agent for these disorders after conversion to nitrosamine-4-(methylnitrosamino)1-(3-pyridyl)-1-butanone (NNK) (Poirier et al., 2004). Prior to NNK conversion, however, nicotine itself exerts harmful effects by interfering with apoptosis. The aim of this study was to characterize the potential anti-apoptotic mechanism of nicotine using established cell lines that were treated with tunicamycin and nicotine. In this report, nicotine can serve as a blocker of apoptosis by enhancing GRP94 expression.

The anti-apoptotic potential of nicotine was characterized in the molecular and physiological perspectives. Despite a difference among established cell lines, apoptosis was considerably inhibited in the presence of nicotine. The difference in inhibiting apoptosis among established cell lines in the present study indicates that different working conditions for nicotine exist among cell lines. Nicotine appears less effective in inhibiting the progression of apoptosis in HepG2 and HEK293 lines. The discrepancy can be explained by dissimilar cellular responses to nicotine among the cell line used in this study. Further study is necessary to understand the diverse apoptotic responses exerted by nicotine.

A significant response to nicotine was quantitated under ERS and ensuing apoptosis. Using combined assays of caspase-3 and TUNEL, we have demonstrated that nicotine inhibits apoptosis caused by ER stress in a concentrationdependent fashion. Apoptosis was significantly inhibited and the level of GRP94 was very high when cells were pre-treated with nicotine. The pre-applied nicotine exerts more negative effects. Nicotine might up-regulate the gene expression or translation of GRP94. Under circumstances, nicotine might serve as antagonist, via increased expression of GRP94, against ERS-induced apoptosis.

Frequent smoking may inhibit ERS-induced apoptosis which contribute a positive effect in removal of damaged cells. Our results are potentially significant considering anti-apoptotic effect of nicotine can be lowered by reducing the expression of GRP94. Plant polyphenols are frequently cited as the effective components to neutralize toxic effect of nicotine (Toda et al., 2004; Malik et al., 2003; Parlermo et al., 2003; Chung et al., 1998). A future study would be meaningful to study natural polyphenol's effect on GRP94 expression during apoptotic development triggered by ERS. Further characterization may be required to understand how nicotine and increment of GRP94 inhibits apoptosis induced under ERS and what factors are involved in the inhibitory outcomes.

During the onset of cancer, apoptosis is very important to remove cancerous cells. This present study thus indicates that nicotine can increase the risk of such cancerous malignancies by inhibiting apoptosis. Along with the proven records of nicotine as apoptotic inducer, the present study indicated that nicotine exerts a dual effect on the development of apoptosis by enhancing GRP94 expression.

\section{ACKNOWLEDGMENTS}

This research was supported by 2007 BioGreen 21 Research Grant from KRDA to DHL. 


\section{REFERENCES}

Blackburn, C. W., Peterson, C. A., Hales, H. A., Carrell, D. T., Jones, K. P., Urry, R. L. and Peterson, C. M. (1994). Nicotine has a direct toxic effect on ovarian function in the immature gonadotropin-stimulated rat. Reprod. Toxicol. 8, 325-331.

Chin, T. Y., Lin H. C., Kuo J. P. and Chueh, S. H. (2007). Dual effect of thapsigargin on cell death in porcine aortic smooth muscle cells. Am J Physiol Cell Physiol, 292, 383 - 395.

Chung, F. L. (1999). The prevention of lung cancer induced by a tobacco-specific carcinogen in rodents by green and black tea. Proc. Soc. Exp. Biol. Med. 220, 244-248.

Chung, F. L., Wang M., Rivenson, A., Iatropoulos, M. J., Reinhardt, J. C., Pittman, B., Ho, C. T. and Amin, S. G. (1998). Inhibition of lung carcinogenesis by black tea in Fischer rats treated with a tobacco-specific carcinogen: caffeine as an important constituent. Cancer Res. 58(18), 4096-4101.

Fassina G., Vene R., Morini M., Minghelli S., Benelli R., Noonan D. M. and Albini A. (2004). Mechanisms of inhibition of tumor angiogenesis and vascular tumor growth by epigallocatechin-3-gallate. Clin Cancer Res. 14, 4865-4873.

Fujiki, H., Suganuma, M., Okabe, S., Sueoka, N., Komori, A., Sueoka, E., Kozu, T., Tada, Y., Suga, K., Imai, K., and Nakachi, K. (1998). Cancer inhibition by green tea. Mutat. Res. 18, 307-310

Gupta, P. C., Murti, P. R., and Bhonsle, R. B. (1996). Epidemiology of cancer by tobacco products and the significance of TSNA. Crit. Rev. Toxicol. 26, 183-198.

Hasseus, B., Wallstrom, M., Osterdahl, B. G., Hirsch J. M. and Jontell M. (1997). Immunotoxic effects of smokeless tobacco on the accessory cell function of rat oral epithelium. Eur. J. Oral Sci. 105, 45-51.

Kalra, R., Singh, S. P., Savage, S. M., Sopori, M. L. and Finch, G.L. (2000). Effects of cigarette smoke on immune response: chronic exposure to cigarette smoke impairs antigen-mediated signaling in T cells and depletes IP3-sensitive $\mathrm{Ca}\left(2^{+}\right)$stores. $J$. Pharmacol. Exp. Ther. 293, 166-171.

Karaskov, E., Scott, C., Zhang, L., Ravazzola, T. M. And Volchuk, A. (2007). Chronic Palmitate But Not Oleate Exposure Induces Endoplasmic Reticulum Stress, Which May Contrib- ute to INS-1 Pancreatic \{beta\}-Cell Apoptosis. Endocrinology, 147, 3398 - 3407.

Lackmann, G. M., Salzberger, U., Tollner, U., Chen, M., Carmella, S. G. and Hecht, S. S. (1999). Metabolites of a tobacco-specific carcinogen in urine from newborns. J. Natl. Cancer Inst. 91, 459-65.

Malik, A., Azam, S., Hadi, N. and Hadi, S. M. (2003). DNA degradation by water extract of green tea in the presence of copper ions: implications for anticancer properties. Phytother. Res. 17, 358-363

Mussmann, R., Geese, M., Harder, F., Kegel, S., Andag, U., Lomow, A., Burk, U., Onichtchouk, D., Dohrmann, C. and Austen, M. (2007). Inhibition of GSK3 Promotes Replication and Survival of Pancreatic Beta Cells. J. Biol. Chem. 282, 12030-12037.

Palermo, C. M., Hernando J. I., Dertinger S. D., Kende A. S. and Gasiewicz T. A. (2003). Identification of potential aryl hydrocarbon receptor antagonists in green tea. Chem Res Toxicol. 16. 865-872.

Poirier, M. C., Santella R. M. and Weston, A. (2000). Carcinogen macromolecular adducts and their measurement Carcinogenesis, 21, 353-359.

Prokopczyk, B., Cox, J. E., Hoffmann, D. and Waggoner S. E. (1997). Identification of tobacco-specific carcinogen in the cervical mucus of smokers and nonsmokers. J. Natl. Cancer Inst. 89, 868-873.

Schuller, H. M., McGavin, M. D., Orloff, M., Riechert, A., and Porter, B. (1995). Simultaneous exposure to nicotine and hyperoxia causes tumors in hamsters. Lab. Invest. 73, 448-456.

Toda, S. (2004). Inhibitory effects of polyphenols in leaves of Artemisia princeps PAMP on protein fragmentation by $\mathrm{Cu}(\mathrm{II})-$ $\mathrm{H}_{2} \mathrm{O}_{2}$ in vitro. $J$ Med. Food. 7, 52-54.

Wynder, E. L., and Muscat, J. E. (1995). The changing epidemiology of smoking and lung cancer histology. Environ. Health Perspect. 103 Suppl. 8, 143-148.

Yung, H. W., Korolchuk, S. Tolkovsky, A. M., Charnock-Jones, D. S. and Burton, G. J. (2007). Endoplasmic reticulum stress exacerbates ischemia-reperfusion-induced apoptosis through attenuation of Akt protein synthesis in human choriocarcinoma cells. FASEB J., 21, 872 - 884. 